International Journal of Pure and Applied Mathematics

Volume 91 No. 3 2014, 305-312

ISSN: 1311-8080 (printed version); ISSN: 1314-3395 (on-line version)

url: http://www.ijpam.eu

doi: http://dx.doi.org/10.12732/ijpam.v91i3.3

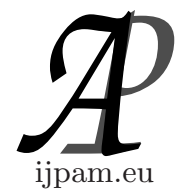

\title{
AFFINE OSSERMAN CONNECTIONS WHICH ARE RICCI FLAT BUT NOT FLAT
}

\author{
Abdoul Salam Diallo ${ }^{1}$, Mouhamadou Hassirou ${ }^{2}$, Issoufou Katambé ${ }^{3}$ \\ ${ }^{1}$ Département de Mathématiques \\ Faculté des Sciences \\ Université Gamal Abdel Nasser \\ B.P. 11 47, Conakry, REPUBLIC OF GUINÉE \\ ${ }^{2,3}$ Département de Mathématiques et Informatique \\ Faculté des Sciences et Technique \\ Université Abdou Moumouni \\ P.O. Box 10 662, Niamey, NIGER
}

Abstract: The present paper deals with the existence of new class of affine Osserman connections which are Ricci flat but not flat.

AMS Subject Classification: 53B05, 53B20, 53C30

Key Words: affine manifold, Osserman connection

\section{Introduction}

Let $(M, \nabla)$ be a $m$-dimensional affine manifold, i.e., $\nabla$ is a torsion free connection on the tangent bundle. Let $\mathcal{R} \nabla(X, Y):=\nabla_{X} \nabla_{Y}-\nabla_{Y} \nabla_{X}-\nabla_{[X, Y]}$ be the associated curvature operator. We define the affine Jacobi operator $J_{\mathcal{R} \nabla}(X): T_{p} M \longrightarrow T_{p} M$ with respect to a vector $X \in T_{p} M$ by

Received: October 23, 2013

(C) 2014 Academic Publications, Ltd. url: www.acadpubl.eu

${ }^{\S}$ Correspondence author 


$$
J_{\mathcal{R} \nabla}(X) Y:=\mathcal{R}^{\nabla}(Y, X) X .
$$

Let $\operatorname{Spect}\left\{J_{\mathcal{R} \nabla}(X)\right\} \subset \mathbb{C}$ be the spectrum of $J_{\mathcal{R} \nabla}(X)$; this is the set of roots of the characteristic polynomial $P_{\lambda}\left[J_{\mathcal{R} \nabla}(X)\right]$. One says that an affine manifold $(M, \nabla)$ is affine Osserman if $\operatorname{Spect}\left\{J_{\mathcal{R}} \nabla(X)\right\}=\{0\}$ for any vector $X$; i.e $\operatorname{Spect}\left\{J_{\mathcal{R} \nabla}(X)\right\}$ is nilpotent.

Affine Osserman manifolds are well-understood in dimension two, due to the fact that an affine manifold is Osserman if and only if its Ricci tensor is skewsymmetric $[4,8]$. The situation is however more involved in higher dimensions where the skew-symmetric is a necessary (but not a sufficient) condition for an affine manifold to be Osserman $[5,6,7]$.

The concept of affine Osserman connection originated from the effort to build up examples of pseudo-Riemannian Osserman manifolds (see $[5,6,9]$ ) via the construction called the Riemannian extensions. This construction, which relates affine and pseudo-Riemannian geometries, assigns to every $m$-dimensional manifold $M$ with a torsion-free affine connection $\nabla$ a pseudo-Riemannian metric $\bar{g}$ of signature $(m, m)$ on the cotangent bundle $T^{*} M$.

Affine Osserman manifolds are of interest not only in affine geometry, but also in the study of pseudo-Riemannian Osserman metrics since they provide some nice examples of Osserman manifolds whose Jacobi operators have nontrivial Jordan normal form and which are not nilpotent $[2,3]$. It has long been a task in this field to build examples of Osserman manifolds which were not nilpotent and which exhibit non-trivial Jordan normal form.

Our paper is organized as follows. Section 1 introduces this topic. In Section 2 we recall some basic definitions and results about affine manifolds. In Section 3 , we study the Osserman condition on two particular affine connections (cf. Theorems 3.1 and 3.2). As applications, one obtains a procedure to build new examples of affine Osserman connections which are Ricci flat but not flat. These families are different from the one we gave in the paper [7] which is not Ricci flat.

\section{Affine Manifolds}

In this section, we give the necessary tools needed to reach our goal. Here are some basic definitions and results about affine manifolds taken from the book [10].

Let $M$ be a 3 -dimensional and $\nabla$ a smooth affine connection. We choose a fixed coordinate domain $\mathcal{U}\left(u_{1}, u_{2}, u_{3}\right) \subset M$. In $\mathcal{U}$, the connection is given by

$$
\nabla_{\partial_{i}} \partial_{j}=\Gamma_{i j}^{k} \partial_{k},
$$


where we denote $\partial_{i}=\left(\frac{\partial}{\partial u_{i}}\right)$ and the functions $\Gamma_{i j}^{k}(i, j, k=1,2,3)$ are called the Christoffel symbols for the affine connection relative to the local coordinate system. We define a few tensor fields associated to a given affine connection $\nabla$. The torsion tensor field $T^{\nabla}$, which is of type $(1,2)$, is defined by

$$
T^{\nabla}(X, Y)=\nabla_{X} Y-\nabla_{Y} X-[X, Y]
$$

The components of the torsion tensor $T^{\nabla}$ in local coordinates are

$$
T_{i j}^{k}=\Gamma_{i j}^{k}-\Gamma_{j i}^{k} \text {. }
$$

If the torsion tensor of a given affine connection $\nabla$ is 0 , we say that $\nabla$ is torsion-free.

The curvature tensor field $\mathcal{R}^{\nabla}$, which is of type $(1,3)$, is defined by

$$
\mathcal{R}^{\nabla}(X, Y) Z:=\nabla_{X} \nabla_{Y} Z-\nabla_{Y} \nabla_{X} Z-\nabla_{[X, Y]} Z \text {. }
$$

The components in local coordinates are

$$
\mathcal{R}^{\nabla}\left(\partial_{k}, \partial_{l}\right) \partial_{j}=\sum_{i} R_{j k l}^{i} \partial_{i}
$$

We shall assume that $\nabla$ is torsion-free. If $\mathcal{R}^{\nabla}=0$ on $M$, we say that $\nabla$ is flat affine connection. It is known that $\nabla$ is flat if and only if around a point there exists a local coordinates system such that $\Gamma_{i j}^{k}=0$ for all $i, j$ and $k$.

We define the Ricci tensor Ric ${ }^{\nabla}$, of type $(0,2)$ by

$$
\operatorname{Ric}^{\nabla}(Y, Z)=\operatorname{trace}\left\{X \mapsto \mathcal{R}^{\nabla}(X, Y) Z\right\} .
$$

The components in local coordinates are given by

$$
\operatorname{Ric}^{\nabla}\left(\partial_{j}, \partial_{k}\right)=\sum_{i} R_{k i j}^{i} .
$$

It is known in Riemannian geometry that the Levi-Civita connection of a Riemannian metric has symmetric Ricci tensor, that is, $\operatorname{Ric}(Y, Z)=\operatorname{Ric}(Z, Y)$. But this property is not true for an arbitrary affine connection which is torsionfree.

\section{Main Results}

In this section we study two families of affine connections and we give the conditions for them to be Osserman. 


\section{Family I}

Theorem 3.1. Let $M$ be a 3-dimensional manifold with torsion free connection given by

$$
\left\{\begin{array}{l}
\nabla_{\partial_{1}} \partial_{1}=f_{1}\left(u_{1}, u_{2}, u_{3}\right) \partial_{2} \\
\nabla_{\partial_{2}} \partial_{2}=f_{2}\left(u_{1}, u_{2}, u_{3}\right) \partial_{3} \\
\nabla_{\partial_{3}} \partial_{3}=f_{3}\left(u_{1}, u_{2}, u_{3}\right) \partial_{1}
\end{array}\right.
$$

Then $(M, \nabla)$ is affine Osserman if and only if the Christoffel symbols of the connection (1) satisfy:

1. $f_{1}=f\left(u_{1}\right), \quad f_{2} \neq f\left(u_{2}\right) e^{\frac{\alpha_{1}}{\alpha_{2}} \int f_{1}\left(u_{1}\right) d u_{1}}$ and $f_{3}=0$.

2. $f_{1}=0, \quad f_{2}=f\left(u_{2}\right)$ and $f_{3} \neq f\left(u_{3}\right) e^{\frac{\alpha_{2}}{\alpha_{3}} \int f_{2}\left(u_{2}\right) d u_{2}}$.

3. $f_{1} \neq f\left(u_{1}\right) e^{\frac{\alpha_{3}}{\alpha_{1}} \int f_{3}\left(u_{3}\right) d u_{3}}, \quad f_{2}=0 \quad$ and $\quad f_{3}=f\left(u_{3}\right)$.

Proof. We denote the functions $f_{1}\left(u_{1}, u_{2}, u_{3}\right), f_{2}\left(u_{1}, u_{2}, u_{3}\right), f_{3}\left(u_{1}, u_{2}, u_{3}\right)$ by $f_{1}, f_{2}, f_{3}$ respectively, if there is no risk of confusion. It is known that the Ricci tensor of any affine Osserman is skew-symmetric. The Ricci tensor of the connection (1) expressed in the coordinates $\left(u_{1}, u_{2}, u_{3}\right)$ takes the form

$$
\text { Ric }=\left(\begin{array}{ccc}
\partial_{2} f_{1} & 0 & 0 \\
0 & \partial_{3} f_{2} & 0 \\
0 & 0 & \partial_{1} f_{3}
\end{array}\right)^{.}
$$

It follows from the expression (2) that we have the following necessary condition for the connection (1) to be Osserman

$$
f_{1}=f\left(u_{1}, u_{3}\right), \quad f_{2}=f\left(u_{1}, u_{2}\right) \quad \text { and } \quad f_{3}=f\left(u_{2}, u_{3}\right) .
$$

Now, a calculation of the Jacobi operators shows that for each vector $X=$ $\sum_{i=1}^{3} \alpha_{i} \partial_{i}$, the associated Jacobi operator is given by

$$
\left(J_{\mathcal{R} \nabla}(X)\right)=\left(\begin{array}{lll}
0 & b_{1} & c_{1} \\
a_{2} & 0 & c_{2} \\
a_{3} & b_{3} & 0
\end{array}\right),
$$

with

$$
b_{1}=\alpha_{3}\left(-\alpha_{1} \partial_{3} f_{1}+\alpha_{3} f_{1} f_{3}\right) \quad c_{1}=\alpha_{2}\left(\alpha_{2} \partial_{1} f_{2}-\alpha_{1} f_{1} f_{2}\right)
$$




$$
\begin{aligned}
& a_{2}=\alpha_{3}\left(\alpha_{3} \partial_{2} f_{3}-\alpha_{2} f_{2} f_{3}\right) \quad c_{2}=\alpha_{1}\left(-\alpha_{2} \partial_{1} f_{2}+\alpha_{1} f_{1} f_{2}\right) \\
& a_{3}=\alpha_{2}\left(-\alpha_{3} \partial_{2} f_{3}+\alpha_{2} f_{2} f_{3}\right) \quad b_{3}=\alpha_{1}\left(\alpha_{1} \partial_{3} f_{1}-\alpha_{3} f_{1} f_{3}\right)
\end{aligned}
$$

It follows from the matrix associated to $J_{\mathcal{R} \nabla}(X)$, that its characteristic polynomial can be written as follows:

$$
P_{\lambda}\left[J_{\mathcal{R} \nabla}(X)\right]=-\lambda^{3}+\left(b_{3} c_{2}+a_{2} b_{1}+a_{3} c_{1}\right) \lambda+\left(a_{3} b_{1} c_{2}+a_{2} b_{3} c_{1}\right) .
$$

It follows that a connection given by (1) is affine Osserman if and only if:

$$
b_{3} c_{2}+a_{2} b_{1}+a_{3} c_{1}=0 \text { and } a_{3} b_{1} c_{2}+a_{2} b_{3} c_{1}=0 .
$$

Since $J_{\mathcal{R} \nabla}(X)=0$, it is easy to see that: $a_{3} b_{1} c_{2}+a_{2} b_{3} c_{1}=0$. Thus $\operatorname{Spect}\left\{J_{\mathcal{R} \nabla}(X)\right\}=\{0\}$ if and only if:

$$
b_{3} c_{2}+a_{2} b_{1}+a_{3} c_{1}=0 .
$$

Let $\frac{a_{3}}{\alpha_{2}}=\frac{-a_{2}}{\alpha_{3}}=A, \quad \frac{-b_{3}}{\alpha_{1}}=\frac{b_{1}}{\alpha_{3}}=B \quad$ and $\quad \frac{c_{2}}{\alpha_{1}}=\frac{-c_{1}}{\alpha_{2}}=C$. The equation (3) becomes:

$$
\alpha_{1}^{2} B C+\alpha_{2}^{2} A C+\alpha_{3}^{2} A B=0 .
$$

Since $\alpha_{i} \neq 0,(i=1,2,3)$, then $A B=A C=B C=0$.

1. Suppose $A=B=0$ and $C \neq 0$. Straightforward calculation gives:

$$
f_{1}=f\left(u_{1}\right), \quad f_{2} \neq f\left(u_{2}\right) e^{\frac{\alpha_{1}}{\alpha_{2}} \int f_{1}\left(u_{1}\right) d u_{1}} \quad \text { and } \quad f_{3}=0 .
$$

2. Suppose $B=C=0$ and $A \neq 0$. Straightforward calculation gives:

$$
f_{1}=0, \quad f_{2}=f\left(u_{2}\right) \quad \text { and } \quad f_{3} \neq f\left(u_{3}\right) e^{\frac{\alpha_{2}}{\alpha_{3}} \int f_{2}\left(u_{2}\right) d u_{2}} .
$$

3. Suppose $A=C=0$ and $B \neq 0$. Straightforward calculation gives:

$$
f_{1} \neq f\left(u_{1}\right) e^{\frac{\alpha_{3}}{\alpha_{1}} \int f_{3}\left(u_{3}\right) d u_{3}}, \quad f_{2}=0, \quad \text { and } \quad f_{3}=f\left(u_{3}\right) .
$$

Example 3.1. Using the affine connection described above (1), one can construct examples of affine Osserman connections. Consider the following connections on $\mathbb{R}^{3}$ given by:

1. $\nabla_{\partial_{1}} \partial_{1}=u_{1}^{2} \partial_{2}, \nabla_{\partial_{2}} \partial_{2}=\left(u_{1}+u_{2}\right) \partial_{3}, \nabla_{\partial_{3}} \partial_{3}=0$;

2. $\nabla_{\partial_{1}} \partial_{1}=0, \nabla_{\partial_{2}} \partial_{2}=u_{2} \partial_{3}, \nabla_{\partial_{3}} \partial_{3}=u_{2} u_{3}^{2}$;

3. $\nabla_{\partial_{1}} \partial_{1}=u_{1} u_{3} \partial_{2}, \nabla_{\partial_{2}} \partial_{2}=0, \nabla_{\partial_{3}} \partial_{3}=e^{u_{3}}$. 


\section{Family II}

Theorem 3.2. Let $M$ be a 3-dimensional manifold with torsion free connection given by

$$
\left\{\begin{array}{l}
\nabla_{\partial_{1}} \partial_{1}=f_{1}\left(u_{1}, u_{2}, u_{3}\right) \partial_{3}, \\
\nabla_{\partial_{2}} \partial_{2}=f_{2}\left(u_{1}, u_{2}, u_{3}\right) \partial_{1}, \\
\nabla_{\partial_{3} \partial_{3}}=f_{3}\left(u_{1}, u_{2}, u_{3}\right) \partial_{2} .
\end{array}\right.
$$

Then $(M, \nabla)$ is affine Osserman if and only if the Christoffel symbols of the connection (4) satisfy:

1. $f_{1} \neq f\left(u_{1}\right) e^{\frac{\alpha_{2}}{\alpha_{1}} \int f_{2}\left(u_{2}\right) d u_{2}}, \quad f_{2}=f\left(u_{2}\right) \quad$ and $\quad f_{3}=0$;

2. $f_{1}=0, \quad f_{2} \neq f\left(u_{2}\right) e^{\frac{\alpha_{3}}{\alpha_{2}} \int f_{3}\left(u_{3}\right) d u_{3}} \quad$ and $\quad f_{3}=f\left(u_{3}\right)$;

3. $f_{1}=f\left(u_{1}\right), \quad f_{2}=0 \quad$ and $\quad f_{3} \neq f\left(u_{3}\right) e^{\frac{\alpha_{1}}{\alpha_{3}} \int f_{1}\left(u_{1}\right) d u_{1}}$.

Remark 3.1. This second family is obtained from the family I by a permutation of indices and are in fact the same properties. Therefore the third family obtained by this permutation gives examples of not Ricci flat Osserman connections (see [7] for details).

Example 3.2. Using the affine connection described above (4), one can construct examples of affine Osserman connections. Consider the following connections on $\mathbb{R}^{3}$ given by:

1. $\nabla_{\partial_{1}} \partial_{1}=u_{1} e^{u_{2}} \partial_{3}, \quad \nabla_{\partial_{2}} \partial_{2}=\frac{a}{u_{2}} \partial_{1}, a \neq 0, \quad \nabla_{\partial_{3}} \partial_{3}=0$;

2. $\nabla_{\partial_{1}} \partial_{1}=0, \quad \nabla_{\partial_{2}} \partial_{2}=u_{2}\left(1+u_{3}^{2}\right) \partial_{1}, \quad \nabla_{\partial_{3}} \partial_{3}=u_{3} \partial_{2} ;$

3. $\nabla_{\partial_{1}} \partial_{1}=\frac{b}{u_{1}} \partial_{3}, b \neq 0, \quad \nabla_{\partial_{2}} \partial_{2}=0, \quad \nabla_{\partial_{3}} \partial_{3}=\frac{c}{u_{1} u_{3}} \partial_{2}, c \neq 0$.

Remark 3.2. Examples 3.1 and 3.2 are important in neutral signature Osserman geometry. For a torsion-free connextion $\nabla$ on $M$, the cotangent bundle $T^{*} M$ may be equipped with a pseudo-Riemannianc metric $g_{\nabla}$ of signature $(m, m)$ : its Riemannian extensions. It is known that, $\left(T^{*} M, g_{\nabla}\right)$ is a pseudo-Riemannian globally Osserman manifold if and only if $(M, \nabla)$ is an affine Osserman manifold. Twisted Riemannian extensions, as a generalization of Riemannian extensions, have been useful in the construction of Kaehler and para-Kaehler Osserman metrics [1]. Calviño-Louzao et al. [2, 3] modify this 
construction to build Osserman Osserman metrics whose Jacobi operators have non-trivial Jordan normal form and which are not nilpotent. It has long been a task in this field to build examples of Osserman manifolds which were not nilpotent and which exhibit non-trivial Jordan normal form.

\section{Acknowledgments}

Research of A. S. Diallo was supported by the RAGAAD. Research of M. Hassirou and I. Katambé was partially supported by the Faculté des Sciences et Techniques de l'Université Abdou Moumouni (Niger). The authors wish to express their thanks to Professor Zibo Garba for discussions and his interest in our work.

\section{References}

[1] A. Bonome, R. Castro, E. García-Rio, L. Hervella, R. Vázquez-Lorenzo, Nonsymmetric Osserman indefinite Kaehler manifolds, Proc. Amer. Math. Soc., 126 (1998), 2763-2769.

[2] E. Calviño-Louzao, E. García-Rio, P. Gilkey, R. Vázquez-Lorenzo, The geometry of modified Riemannian extensions, Proc. R. Soc., A, 465 (2009), 2023-2040.

[3] E. Calviño-Louzao, E. García-Rio, P. Gilkey, R. Vázquez-Lorenzo, Higherdimensional Osserman metrics with non-nilpotent Jacobi operators, Geom. Dedicata, 156 (2012), 151-163.

[4] A.S. Diallo, Affine Osserman connections on 2-dimensional manifolds, Afric. Dispora J. Math., 11, No. 1 (2011), 103-109.

[5] A.S. Diallo, The Riemann extension of an affine Osserman connection on 3-dimensional manifold, Global Journal of Advanced Research on Classical and Modern Geometries, 2, No. 2 (2013), 69-75.

[6] A.S. Diallo, M. Hassirou, Examples of Osserman metrics of $(3,3)$-signature, J. Math. Sci. Adv. Appl., 7, No. 2 (2011), 95-103.

[7] A.S. Diallo, M. Hassirou, Two families of affine Osserman connections on 3-dimensional manifolds, Afr. Diaspora J. Math., 14, No. 2 (2012), 178186. 
[8] E. García-Rio, D.N. Kupeli, M.E. Vázquez-Abal, R. Vázquez-Lorenzo, Affine Osserman connections and their Riemannian extensions, Differential Geom. Appl., 11 (1999), 145-153.

[9] E. García-Rio, D.N. Kupeli, R. Vázquez-Lorenzo, Osserman Manifolds in Semi-Riemannian Geometry, Lectures Notes in Mathematics, 1777, Springer-Verlag, Berlin (2002).

[10] K. Nomizu, T. Sasaki, Affine Differential Geometry, Cambridge University Press, 111 (2008). 